\title{
MicroRNA-449 targets histone deacetylase 1 to regulate the proliferation, invasion, and apoptosis of synovial fibroblasts in rheumatoid arthritis
}

\author{
Jiangtao Guo ${ }^{1,2,3,4 \#}$, Xuqing Cao ${ }^{2,3,5 \#}$, Wei Zhao ${ }^{2,4 \#}$, Hong Zhu ${ }^{6}$, Xiaoli Ma ${ }^{1}$, Chunfang $\mathrm{Hao}^{1}$, Lili $\mathrm{Wu}^{1}$, \\ Mingzhu Zhang ${ }^{1}$, Yashan Yang ${ }^{1}, J^{\prime}$ gtian Zhao ${ }^{1,2}$, Kunting Chen ${ }^{1,2}$, Zhe Yin ${ }^{1,2}$ \\ ${ }^{1}$ Department of Rheumatology and Immunology, People's Hospital of Ningxia Hui Autonomous Region, Yinchuan, China; ${ }^{2}$ The Third Affiliated \\ Hospital, Ningxia Medical University, Yinchuan, China; ${ }^{3}$ The First Affiliated Hospital of Northwest Minzu University, Yinchuan, China; ${ }^{4}$ Ningxia \\ Key Laboratory of Precision Medicine for Autoimmune Diseases, Yinchuan, China; ${ }^{5}$ Department of Neurology, People's Hospital of Ningxia Hui \\ Autonomous Region, Yinchuan, China; ${ }^{\circ}$ Department of Rheumatology, General Hospital of Ningxia Medical University, Yinchuan, China \\ Contributions: (I) Conception and design: J Guo, X Cao, W Zhao; (II) Administrative support: H Zhu, J Zhao; (III) Provision of study materials or \\ patients: J Guo, X Cao, X Ma, C Hao, L Wu, M Zhang, Y Yang, K Chen, Z Yin; (IV) Collection and assembly of data: All authors; (V) Data analysis \\ and interpretation: J Guo, X Cao, W Zhao, H Zhu, J Zhao, X Ma, C Hao, L Wu; (VI) Manuscript writing: All authors; (VII) Final approval of \\ manuscript: All authors. \\ \#These authors contributed equally to this work. \\ Correspondence to: Xuqing Cao. Department of Neurology, People's Hospital of Ningxia Hui Autonomous Region, 301 Zhengyuan North Street, \\ Jinfeng District, Ningxia Hui Autonomous Region, Yinchuan, China. Email: 13909592466@163.com.
}

Background: Rheumatoid arthritis (RA) is a chronic joint disease. The study aimed to explore the effects of microRNA (miR)-449 and histone deacetylase 1 (HDAC1) on the proliferation, invasion, and apoptosis of synovial fibroblasts in rheumatoid arthritis.

Methods: Synovial tissue was collected from 20 patients with RA and 20 patients with osteoarthritis (OA) who underwent joint replacement surgery. RA synovial fibroblasts (RASFs) and OA synovial fibroblasts (OASFs) were isolated and cultured. Real-time quantitative PCR was used to detect the expression levels of miR-449 and HDAC1 in synovial tissues and cells. Western blot was performed to detect the cellular expression levels of HDAC1 protein, and apoptosis and invasion-related proteins. The proliferation, invasion, and apoptosis of RASFs were detected by MTT assay, Transwell assay, and flow cytometry. The dual-luciferase reporter gene was used to test the targeting relationship between inflammatory miR-449 and HDAC1.

Results: Compared with normal synovial tissue and OASFs, the levels of HDAC1 messenger RNA in RA synovial tissue and RASF cells were significantly increased $(\mathrm{P}<0.01)$, while the expression levels of miR-449 were significantly decreased $(\mathrm{P}<0.01)$. The dual-luciferase reporter gene experiment confirmed that miR449 could specifically bind to the 3 ' untranslated region of HDAC1 to inhibit its luciferase activity $(\mathrm{P}<0.05)$. HDAC1 inhibition or miR-449 overexpression significantly inhibited the proliferation and invasion of RASFs $(\mathrm{P}<0.001)$, while inducing their apoptosis $(\mathrm{P}<0.001)$. HDAC1 overexpression reversed the biological effects of miR-449 on RASFs $(\mathrm{P}<0.001)$.

Conclusions: miR-449 inhibits the proliferation and invasion of RASFs and induces their apoptosis by targeting HDAC1, thereby exerting a protective effect against RA.

Koywords: MicroRNA; miR-449; histone deacetylase 1 (HDAC1); rheumatoid arthritis (RA); osteoarthritis

Submitted May 14, 2021. Accepted for publication Jun 24, 2021.

doi: 10.21037/apm-21-1383

View this article at: https://dx.doi.org/10.21037/apm-21-1383

(c) Annals of Palliative Medicine. All rights reserved. 


\section{Introduction}

Rheumatoid arthritis (RA) is a chronic systemic immune disease characterized by synovitis (1). The main symptom of RA is joint erosion, which can cause joint stiffness, swelling, and pain. RA affects the joint activity of patients in the short term, but without active and effective treatment, it can eventually lead to lung and heart complications, and even lifelong disability. At present, about $0.5 \%$ of the global population suffers from this disease (2). Further study of RA is urgently called for due to the huge economic and social burden the disease places on patients and their families.

Histone deacetylase 1 (HDAC1) has been found to be closely related to the occurrence and development of RA. HDAC1 affects the differentiation of osteoclast precursor cells, the secretion of multiple signaling pathways and a variety of inflammatory cytokines. HDAC1 interferes with the differentiation, maturation and apoptosis of osteoclasts, and promotes the occurrence and development of RA bone destruction. Hawtree et al. indicated that the expression of HDAC1 in RA synovial fibroblasts (RASFs) was significantly higher than that in osteoarthritis (OA) synovial fibroblasts (OASFs). They demonstrated that knockdown of HDAC1 could inhibit the proliferation, migration, and invasion of RASFs in vitro, and that inhibiting HDAC1 could significantly reduce joint swelling and lessen cartilage and joint damage in an RA animal model (3). Göschl et al. showed that the development of RA was inhibited in mice with HDAC1-specific T-cell deletion. They observed that MS-275, an HDAC1 inhibitor, had a good therapeutic effect on mice with RA, which suggested the potential of HDAC1 as a target for the treatment of RA (4). Another study illustrated that many epigenetic changes are involved in the occurrence of RA (5).

So far, research on RA epigenetics has mainly focused on microRNA ( $\mathrm{miRNA} / \mathrm{miR}$ ), with the abnormal expression of many miRNAs having been found in the tissues, cells, and serum of patients with RA. For instance, Evangelatos et al. reported that serum miR-22 and miR-103a levels can serve as biomarkers to predict the RA-prone population (6). A recent study by Wang et al. showed that miR-449 can improve gouty arthritis through its regulation of NLRP3 inflammasome activation (7). Previous studies have established that HDAC1 is a downstream target gene of miR-449, thus suggesting that miR-449 may affect the development of RA by regulating HDAC1 expression. Therefore, the present study aimed to explore the effects of the miR-449/HDAC1 molecular axis on RA development in vitro. We present the following article in accordance with the MDAR reporting checklist (available at https://dx.doi. org/10.21037/apm-21-1383).

\section{Methods}

\section{Reagents}

Fetal bovine serum (FBS), penicillin-streptomycin solution were purchased from Gibco (New York, USA). Dulbecco's Modified Eagle Medium (DMEM), a Lipofectamine RNAMAX transfection kit, a TRIzol kit and TaqMan microRNA analysis kit were purchased from Invitrogen (Carlsbad, California, USA). An RNA reverse transcription kit was purchased from Thermo Scientific (MA, USA). An Annexin V-FITC/PI kit was purchased from Beijing Baosai Biotechnology Co., Ltd (Beijing, China). RIPA lysis buffer a bicinchoninic acid (BCA) assay kit, and a chemiluminescence kit were purchased from Biyuntian Institute of Biotechnology (Shanghai, China). All primary and secondary antibodies used in our experiments were purchased from Abcam (Cambridge, UK). The plasmid pDL3 was purchased from Promega (WI, USA).

\section{Patients}

Synovial tissues were collected from 20 patients with RA who underwent surgery in our hospital between January 2019 and January 2021. The diagnoses of the 20 patients with RA met the American Rheumatic Association's diagnostic criteria for RA. There were 12 male and 8 female patients, who had an average age of $50.5 \pm 3$ years old. Normal synovial tissues (normal group) were also collected from patients with $\mathrm{OA}$ who underwent joint catheterization in our hospital, including 14 males and 6 females, with an average age of $51.3 \pm 53.8$ years old. There was no significant difference in sex or age between the two groups $(\mathrm{P}<0.05)$. All procedures performed in this study involving human participants were in accordance with the Declaration of Helsinki (as revised in 2013). The study was approved by the Ethics Committee of People's Hospital of Ningxia Hui Autonomous Region (No.: 2020-KY-107) and informed consent was taken from all the patients.

\section{Cell culture}

The synovial tissues from the patients were cut up under aseptic conditions. The minced tissue was digested with 
Table 1 Primer sequences

\begin{tabular}{ll}
\hline Gene & Sequence (F: Forward; R: Reverse) \\
\hline HDAC1 & F: 5'-GATTCCTCTCCACACCTGACTTC-3' \\
& R: 5'-TGTTGCCAGAGACGAAGTGGA-3' \\
GAPDH & F: 5'-GAGTCAACGGATTTGGTCGTATTG-3' \\
& R: 5'-CCTGGAAGATGGTGATGGGATT-3' \\
miR-449 & F: 5'-AGGGTGGCAGTGTATTGTTA-3' \\
& R: 5'-GAGAGGAGAGGAAGAGGGAA-3' \\
U6 & F: 5'-CTCGCTTCGGCAGCACA-3' \\
&
\end{tabular}

$0.25 \%$ trypsin and centrifuged $(3,000 \times \mathrm{g})$ for $15 \mathrm{~min}$, and the pellet was collected. And then add the collected precipitate to DMEM complete cell culture medium containing $10 \%$ fetal bovine serum (FBS). The cells were incubated at $37{ }^{\circ} \mathrm{C}$ and $5 \% \mathrm{CO}_{2}$ to make them adhere to the wall of plate. After the removal of non-adherent cells, the adherent synovial fibroblasts were collected by trypsin digestion for 2 hours. Third- to seventh-generation cells were obtained for subsequent experiments.

\section{Cell transfection}

MiR-Negative Control (NC), miR-449 mimics, si-NC, and si-HHAC1 (sequence: 5'-CAGCGACUGUUUG AGAACC-3') were synthesized and purified by Gene Pharma. HDAC1 complementary DNA (cDNA) was inserted into pcDNA3.1 vector to construct HDAC1 overexpression plasmid (pcDNA-HDAC1). At 24 hours before transfection, miR-NC, miR-449 mimics, si-NC, si-HDAC1, pcDNA-NC, and pcDNA-HDAC1 were transfected into RASFs following the instructions supplied with the Lipofectamine RNAMAX transfection kit. After transfection, the cells were incubated for a further 48 hours and digested with trypsin.

\section{Quantitative real-time polymerase chain reaction}

Total RNA was extracted from synovial tissues and cells using TRIzol reagent, and a reverse transcription kit was used to reverse transcribe RNA into cDNA. The expression levels of HDAC1 (with GAPDH as an internal reference) and miR-449 (with U6 as an internal reference) in cells were detected in strict accordance with the instructions supplied with the TaqMan microRNA detection kit. The $2^{-\Delta \Delta \mathrm{Ct}}$ method was used to calculate the relative expression levels of HDAC1 messenger RNA (mRNA) and miR449. The primer sequences used are shown in Table 1. The experiment was repeated 3 times for each group.

\section{Western blot analysis}

Intracellular protein was collected with RIPA lysis buffer, and the BCA kit was employed to determine the protein concentration. The protein was transferred to a $10 \%$ polyvinylidene fluoride (PVDF) membrane. After protein loading $(50 \mu \mathrm{g})$, the PVDF membrane was blocked in $5 \%$ skim milk for 1 hour and incubated with primary antibody $(1: 1,000)$ at $4{ }^{\circ} \mathrm{C}$ overnight. The membrane was then subjected to a second round of incubation, with horseradish peroxidase-labeled Goat anti-rabbit IgG at room temperature for 2 hours. Finally, the protein bands were visualized with a chemiluminescence kit and photographed, and quantification analysis was performed. The experiment was repeated 3 times for each group.

\section{MTT assay}

Cells were digested in trypsin and resuspended in DMEM containing $10 \%$ FBS. The cells inoculated into 96 well plate at a density of $1 \times 10^{5}$, and then incubated at $37^{\circ} \mathrm{C}$ and $5 \% \mathrm{CO}_{2}$ for $0,6,12,24$, and 48 hours. After the serumcontaining medium had been removed, fresh serum-free medium and $15 \mu \mathrm{L}$ methyl thiazolyl tetrazolium $(5 \mathrm{ng} / \mathrm{mL})$ was added to each well, which was followed by 4-hour incubation. After the addition of $150 \mu \mathrm{L}$ dimethyl sulfoxide to each well, the absorbance (OD) value was determined at $450 \mathrm{~nm}$ with a microplate reader.

\section{Cell invasion assay}

Cells were seeded at a density of $1 \times 10^{5}$ into the upper chamber of a Transwell with DMEM-containing medium $(100 \mu \mathrm{L})$. DMEM-containing medium $(600 \mu \mathrm{L})$ was added into the lower chamber of the Transwell. After 6 hours' incubation at $37^{\circ} \mathrm{C}$ and $5 \% \mathrm{CO}_{2}$, the cells in the upper chamber that had not invaded were removed. The cells in the lower chamber were fixed and stained with $0.1 \%$ crystal violet. Three randomly selected fields were photographed and counted. The experiment was repeated 3 times for each group. 

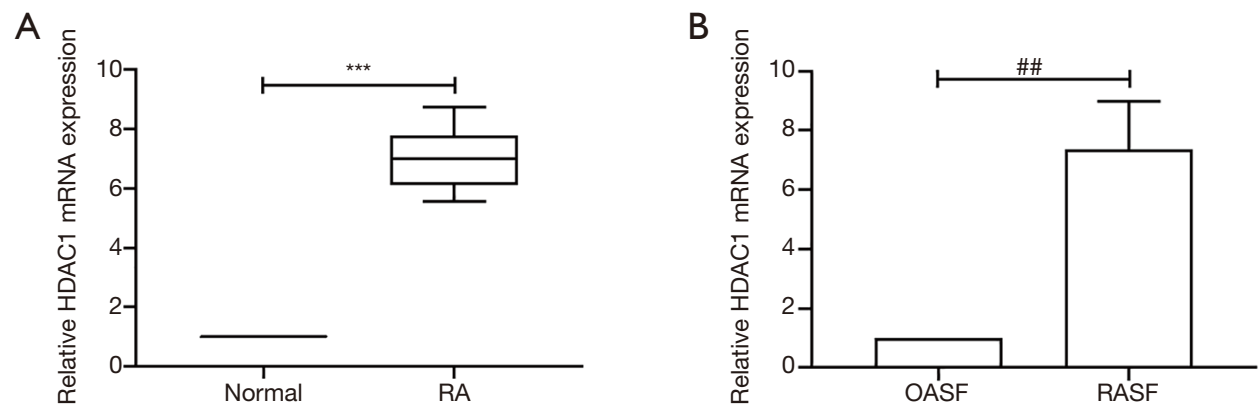

Figure 1 HDAC1 mRNA expression in synovial tissue and cells of the joint was detected by real-time quantitative PCR. (A) Expression of HDAC1 mRNA in joint synovial tissues; (B) expression of HDAC1 mRNA in cells. ${ }^{* * *} \mathrm{P}<0.001$, compared with normal tissues ( $\mathrm{n}=20$ ); ${ }^{\#} \mathrm{P}<0.01$, compared with OASFs ( $\mathrm{n}=3$ ). HDAC1, histone deacetylase 1; OASFs, osteoarthritis synovial fibroblasts; RASFs, RA synovial fibroblasts.

\section{Cell apoptosis assay}

Cells were seeded into 6 -well plates at a density of $1 \times 10^{5}$. Flow cytometry was used to determine the apoptotic rate of the cells in each well in adherence with the instructions supplied with the Annexin V-FITC/PI kit. The experiment was repeated 3 times for each group.

\section{Double-luciferase reporter gene experiment}

Cells were seeded into 24-well plates at a density of $1 \times 10^{5}$. Then, cells were transfected with pGL3-HDAC1 3 ' untranslated region (UTR) wild-type (HDAC1 3'UTR WT) or mutant (HDAC1 3'-UTR MUT) plasmids and miR-NC or miR-449 mimics. After 48-hour transfection, the dual-luciferase reporter system was used to detect the luciferase activities of fireflies and sea kidneys. The experiment for each group was repeated 3 times.

\section{Statistical analysis}

SPSS 25.0 statistical software developed by IBM (NY, USA) was used for the experimental data analysis, and GraphPad Prism version 8.2, developed by GraphPad Software (CA, USA), was employed to draw the statistical charts. Pearson's correlation was used to analyze the correlation between miR-449 and HDAC1 mRNA expression in synovial tissue. The data were presented as mean \pm standard deviation $(\bar{x} \pm s)$. Differences between the two groups were compared by $t$ tests. $\mathrm{P}<0.05$ showed that a difference between the two groups was statistically significant.

\section{Results}

\section{HDAC1 is upregulated in RA synovial tissue and RASFs}

The expression level of HDAC1 mRNA in RA synovial tissues was significantly higher than that in normal tissues $(\mathrm{P}<0.001)$, and it was also significantly increased in RASFs compared to OASFs $(\mathrm{P}<0.01$, Figure 1).

\section{Knockdown of HDAC1 inbibits the proliferation and invasion of RASFs and induces their apoptosis}

RASFs were transfected with si-NC or si-HDAC1. RASF proliferation, invasion, and apoptosis were detected at 48 hours after transfection. The levels of HDAC1 protein and mRNA were significantly decreased in RASFs transfected with si-HDAC1 $(\mathrm{P}<0.001$, Figure $2 A, B, C)$. After si-HDAC1 transfection, RASF proliferation was inhibited considerably $(\mathrm{P}<0.001$, Figure $2 D)$, while the apoptotic rate of RASFs was significantly increased $(\mathrm{P}<0.001$, Figure $2 E, F)$. Further, the protein levels of c-caspase-3, c-caspase-9, and Bax were increased, while that of Bcl-2 was decreased $(\mathrm{P}<0.01$, Figure $2 G, H)$. Additionally, the number of invading RASFs was significantly decreased $(\mathrm{P}<0.001$, Figure $2 I, 7)$, and the protein expression levels of MMP-2 and MMP-9 were significantly reduced $(\mathrm{P}<0.001$, Figure $2 K, L)$.

\section{miR-449 directly targets HDAC1}

The expression level of miR-449 in synovial tissue was significantly decreased in RA tissue compared to normal tissue $(\mathrm{P}<0.001$, Figure $3 A)$, similarly, it was lower in RASFs than in OASFs $(\mathrm{P}<0.01$, Figure $3 B)$. Correlation 
A

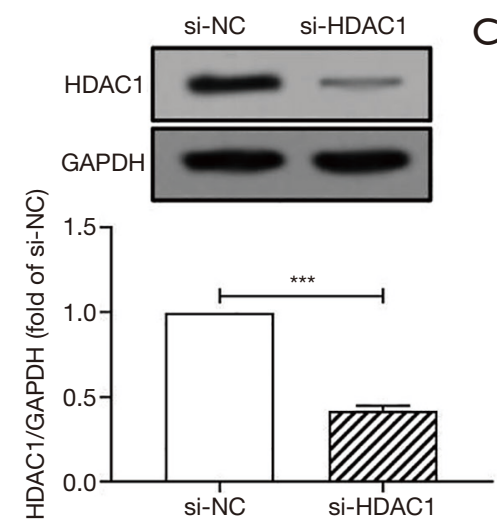

$\mathrm{E}$

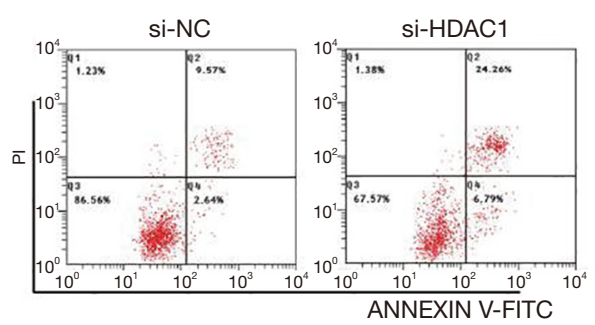

$\mathrm{H}$

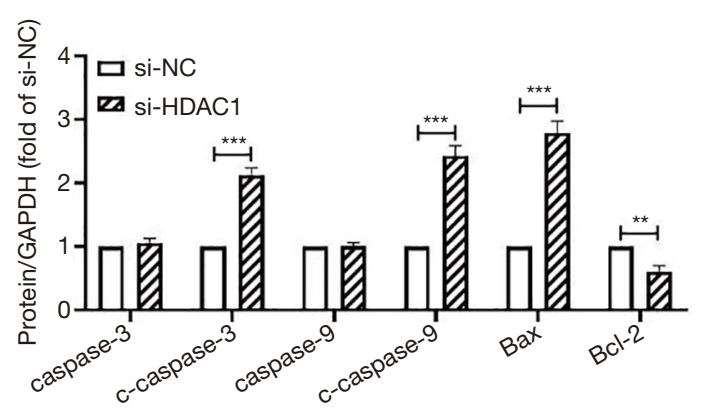

J

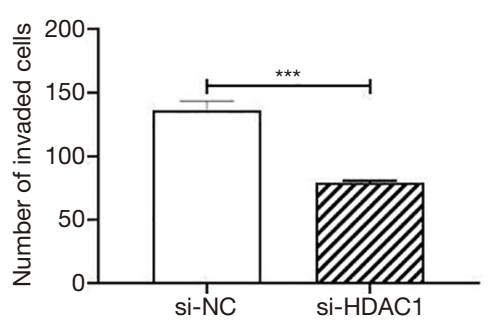

$\mathrm{F}$

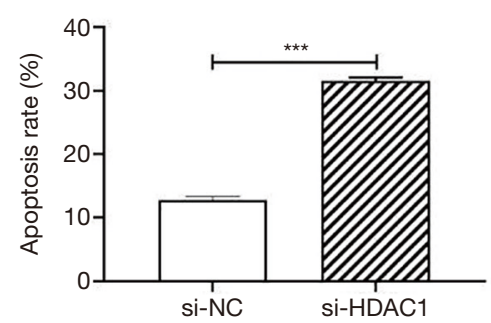

I

K

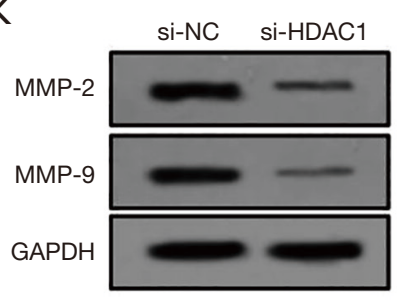

D
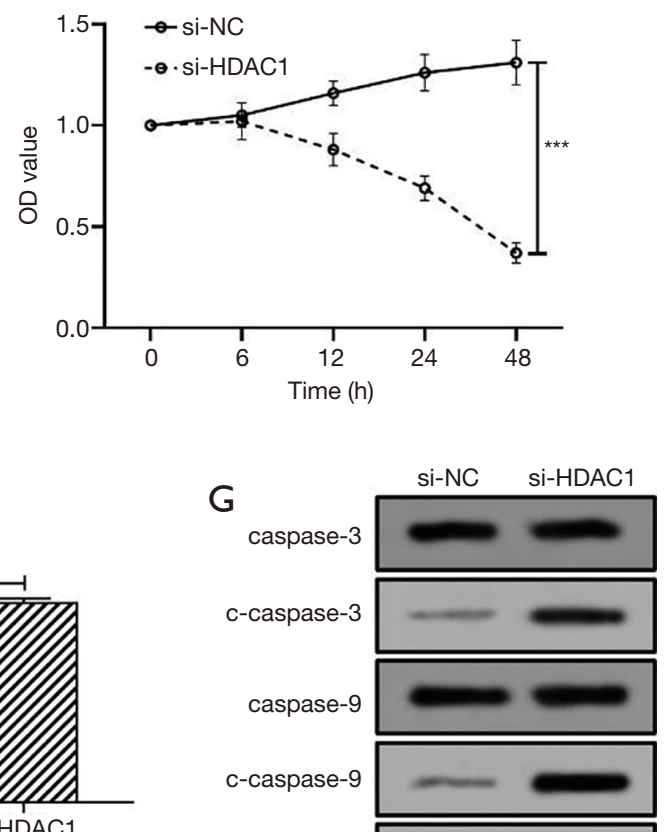

Bax

$\mathrm{Bcl}-2$ si-HDAC1 GAPDH
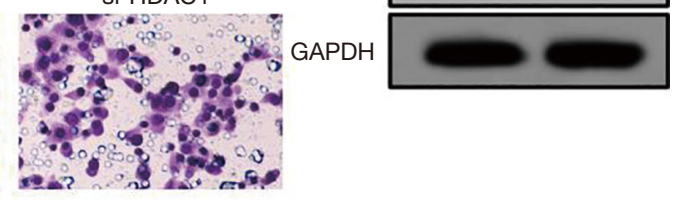

$L \sum_{\frac{i}{\omega}}^{\widehat{O}} 1.57$ ㅁ si-NC

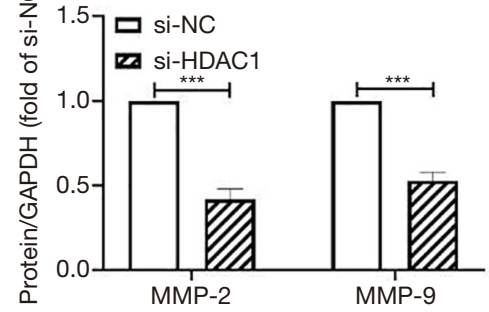

Figure 2 Effects of inhibiting the expression level of HDAC1 on RASF cells. (A,B) HDAC1 protein expression in RASFs; (C) HDAC1 mRNA expression levels in RASFs; (D) the proliferation ability of RASFs; (E,F) the apoptotic rate of RASFs; (G,H) the expression levels of apoptosis-related proteins in RASFs; (I,J) the invasion ability of RASFs (magnification, 40×; dyeing method: crystal violet); (K,L) the expression levels of invasion-related proteins in RASFs. ${ }^{* *} \mathrm{P}<0.01$ and ${ }^{* * *} \mathrm{P}<0.001$, compared with si-NC group ( $\mathrm{n}=3$ ). HDAC1, histone deacetylase 1; RASF, rheumatoid arthritis synovial fibroblast.

analysis uncovered a negative correlation between HDAC1 mRNA expression and miR-449 expression in RA synovial tissue $(\mathrm{P}<0.001$, Figure $3 C)$. StarBase prediction results showed that miR-449 and HDAC1 had partial targeted binding sequences (Figure 3D). In RASFs, overexpression of miR-449 significantly inhibited the luciferase activity 
A

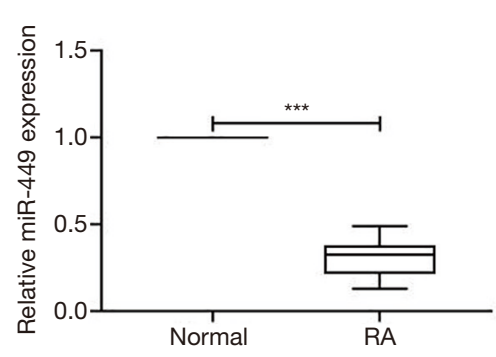

B

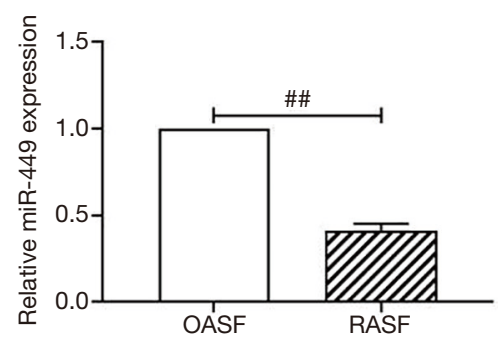

C

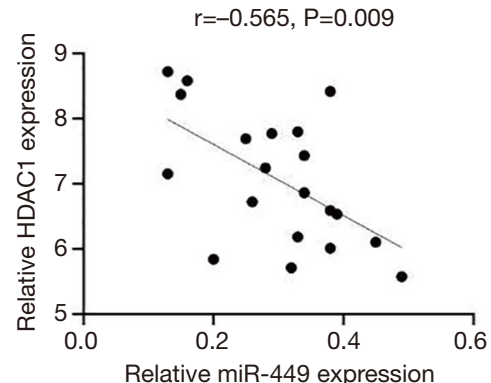

$\mathrm{E}$

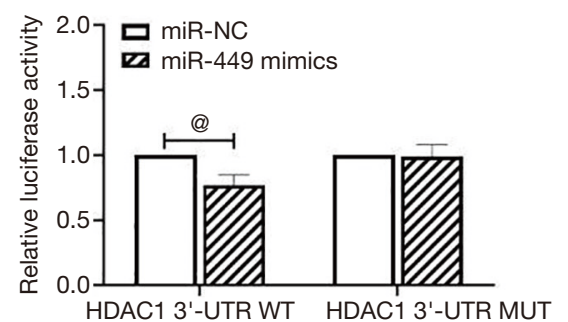

$\mathrm{H}$

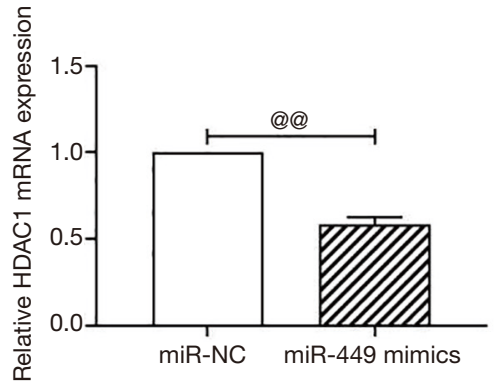

Figure 3 miR-449 directly targets HDAC1. (A,B) miR-449 expression level in RA synovial tissue and RASFs; (C) correlation between the expression levels of HDAC1 mRNA and miR-449; (D) target binding sequence of miR-449 and HDAC1; (E) dual-luciferase activity in RASFs; (F,G) HDAC1 protein expression levels in RASFs; (H) HDAC1 mRNA expression levels in RASFs. ${ }^{* * *} \mathrm{P}<0.001$, compared with normal tissue ( $\mathrm{n}=20) ;{ }^{\# \#} \mathrm{P}<0.01$, compared with OASFs $(\mathrm{n}=3) ;{ }^{\circledR} \mathrm{P}<0.05,{ }^{\circledR} \mathrm{P}<0.01$, and ${ }^{\circledR}{ }^{\circledR} \mathrm{P}<0.001$, compared with miR-NC group $(\mathrm{n}=3)$. HDAC1, histone deacetylase 1; RASF, rheumatoid arthritis synovial fibroblast.

of the HDAC1 3'UTR WT plasmid ( $<<0.05$, Figure 3E), and significantly inhibited the expression of HDAC1 at the protein $(\mathrm{P}<0.001$, Figure $3 F, G)$ and mRNA $(\mathrm{P}<0.01$, Figure $3 H$ ) levels.

\section{Overexpression of miR-449 inbibits the proliferation and invasion of RASFs and induces their apoptosis}

After 48 hours of transfection with miR-449 mimics, RASFs showed significantly increased expression levels of miR-449 $(\mathrm{P}<0.01$, Figure $4 A)$, but their proliferation was significantly inhibited $(\mathrm{P}<0.001$, Figure $4 B)$. The apoptotic rate of RASFs was also significantly increased $(\mathrm{P}<0.001$, Figure $4 C, D)$. Also following transfection, the protein levels of c-caspase-3, c-caspase-9, and Bax in RASFs were elevated, while that of $\mathrm{Bcl}-2$ was decreased $(\mathrm{P}<0.01$, Figure $4 E, F)$. Additionally, the number of invading RASFs was significantly reduced $(\mathrm{P}<0.001$, Figure $4 G, H)$, and the protein levels of MMP-2 and MMP-9 were significantly decreased $(\mathrm{P}<0.001$, Figure 4I, 7$)$.

\section{Overexpression of HDAC1 reverses the biological effects of miR-449 on RASFs}

RASFs were co-transfected with miR-449 mimics and pcDNA-HDAC1. Transfection of pcDNA-HDAC1 significantly reversed the inhibitory effect of miR449 on HDAC1 expression at the protein $(\mathrm{P}<0.001$, Figure $5 A, B)$ and mRNA $(\mathrm{P}<0.001$, Figure $5 C)$ levels. Transfection with pcDNA-HDAC1 significantly 

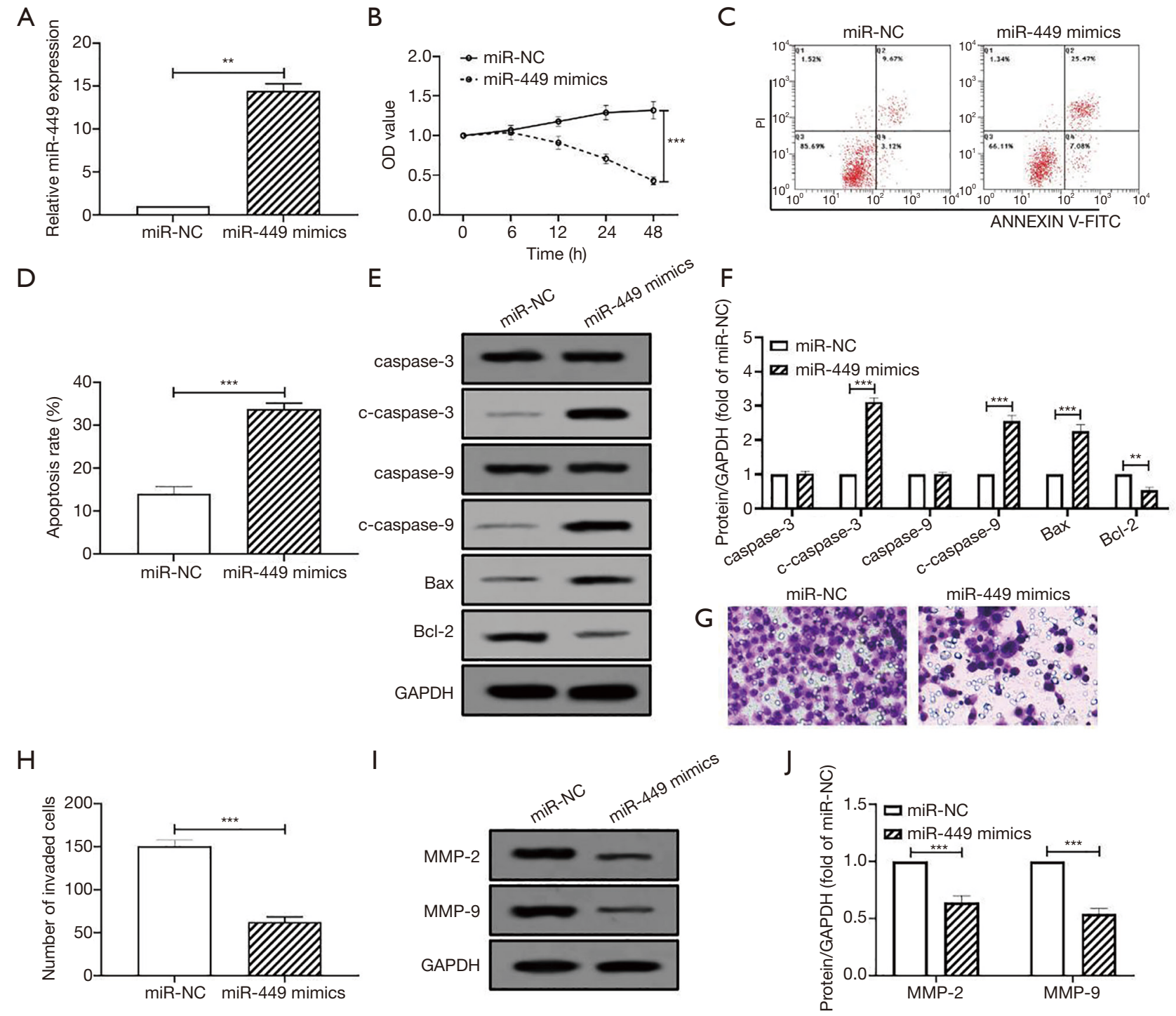

Figure 4 Effects of miR-449 overexpression on RASFs. (A) miR-449 expression levels in RASFs; (B) the proliferation ability of RASFs; (C,D) the apoptotic rate of RASFs; (E,F) the expression levels of apoptosis-related proteins in RASFs; $(\mathrm{G}, \mathrm{H})$ the invasion ability of RASFs (magnification, 40x; dyeing method: crystal violet); (I,J) the expression levels of invasion-related proteins in $\mathrm{RASFs}$. ${ }^{* *} \mathrm{P}<0.01$ and *** $\mathrm{P}<0.001$, compared with miR-NC group $(\mathrm{n}=3)$. RASFs, rheumatoid arthritis synovial fibroblasts.

enhanced the cell proliferation $(\mathrm{P}<0.001$, Figure 5D) and significantly inhibited the apoptosis $(\mathrm{P}<0.001$, Figure $5 E, F)$ of mir-449-overexpressing RASFs, thus reducing the protein levels of c-caspase-3, c-caspase-9, and Bax, while increasing that of Bcl-2 $(\mathrm{P}<0.001$, Figure $5 G, H)$. Furthermore, transfection with pcDNAHDAC1 reversed the inhibitory effect of miR-449 overexpression on the invasive ability of RASFs $(\mathrm{P}<0.001$, Figure 5I,7), and the expression levels of MMP-2 and MMP-
9 showed significant increases $(\mathrm{P}<0.001$, Figure $5 K, L)$.

\section{Discussion}

RASF is the main effector cell of rheumatoid arthritis. The proliferation and migration ability of RASF cells in RA patients increases, and the level of apoptosis decreases. In the pathogenesis of RA, RASF cells are activated and release a series of inflammatory factors to mediate the 
A

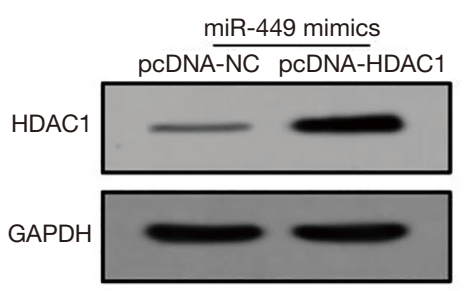

D

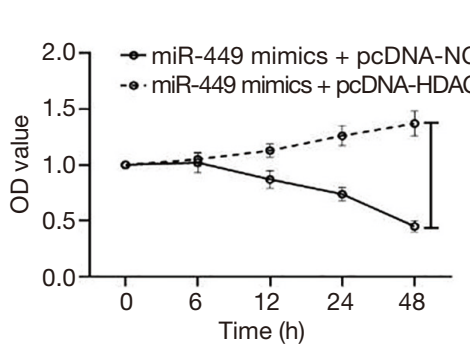

G

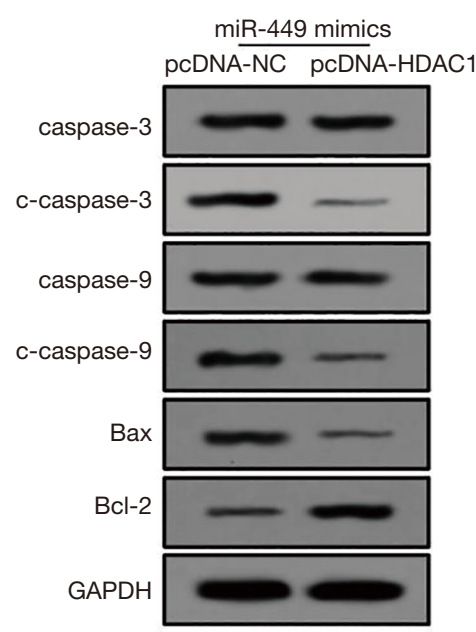

J

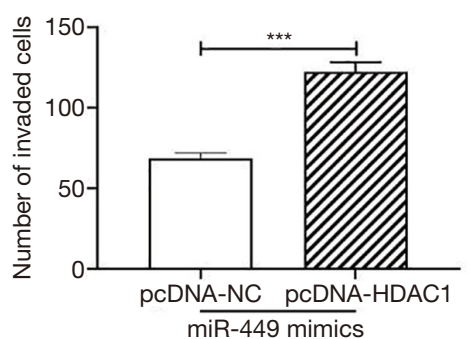

B

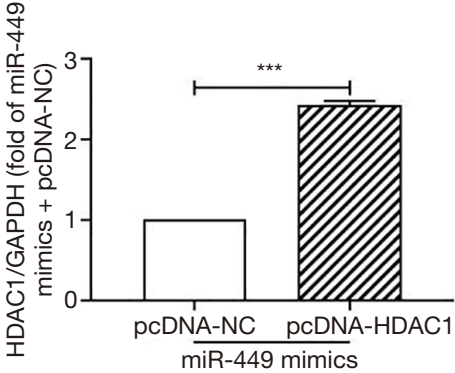

$E$

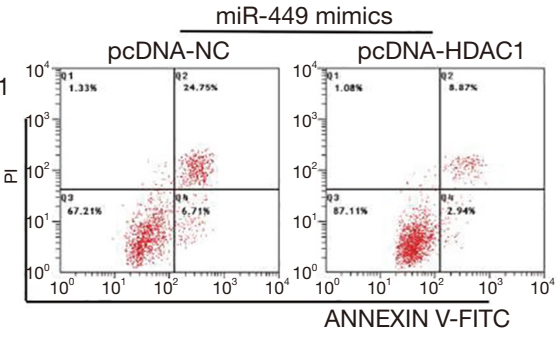

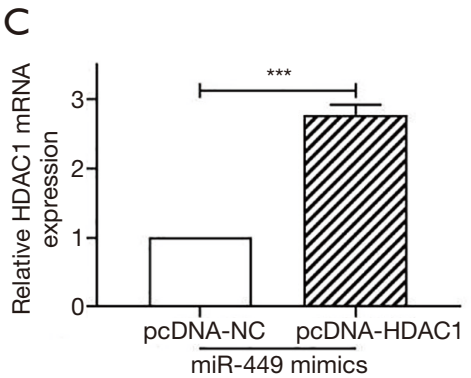

$\mathrm{F}$

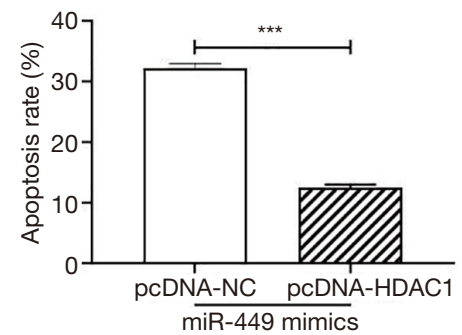

$\mathrm{H}$

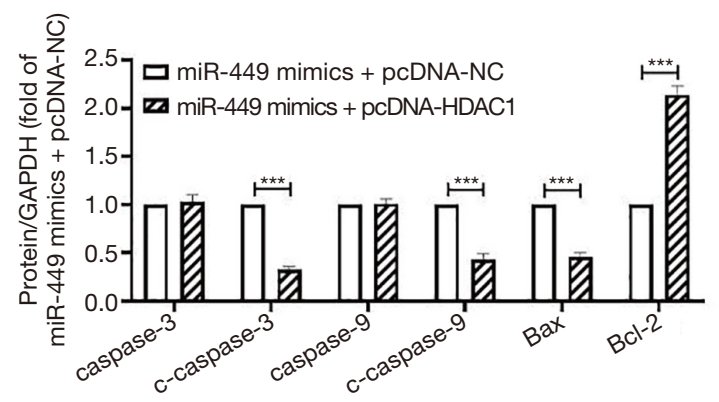

I

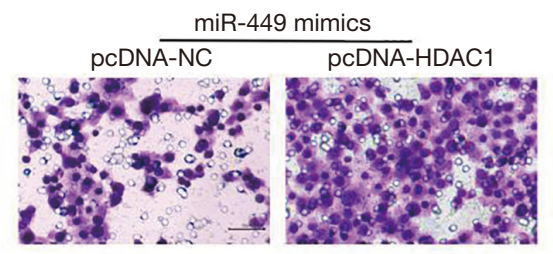

K

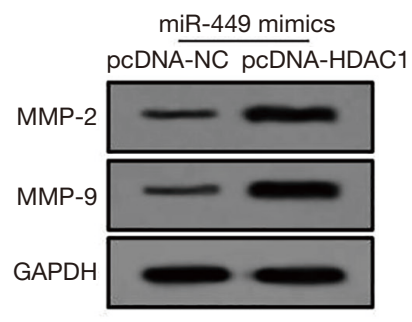

$\mathrm{L}$

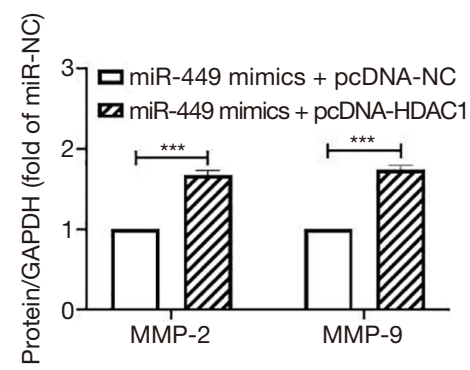

Figure 5 Effects of overexpression of HDAC1 and miR-449 on RASFs. (A,B) HDAC1 protein expression in RASFs; (C) HDAC1 mRNA expression in RASF cells; (D) the proliferation ability of RASFs; (E,F) the apoptotic rate of RASFs; (G,H) expression levels of apoptosisrelated proteins in RASFs; (I,J) the invasion ability of RASFs (magnification, 40x; dyeing method: crystal violet); (K,L) expression levels of invasion-related proteins in RASFs. ${ }^{* * *} \mathrm{P}<0.001$, compared with miR-449 mimics + pcDNA-NC group ( $\left.\mathrm{n}=3\right)$. HDAC1, histone deacetylase 1 ; RASFs, rheumatoid arthritis synovial fibroblasts. 
inflammatory response, causing the destruction of bone tissue and cartilage tissue (3). HDAC1 has been shown to be able to directly or indirectly regulate bone cell formation and resorption (8). Huang et al. indicated that HDAC1 could promote transforming growth factor by inhibiting Wnt activity, thus promoting early chondrogenesis mediated by tumor necrosis factor- $\beta 1$ (9). Furthermore, the research of Miao et al. illustrated that Wnt signaling pathway is critical to RA occurrence and development (10). However, whether HDAC1 can affect the Wnt signaling pathway in RA tissues and cells has yet to be reported. In the present study, we found that HDAC1 expression in RA synovial tissue and fibroblasts was significantly increased. Inhibition of HDAC1 expression significantly minimized the proliferation and invasion of RASFs while promoting their apoptosis, indicating HDAC1 to play an important role in the pathogenesis of RA.

In animals and plants, miRNAs are involved in various biological processes, such as cell proliferation, invasion, apoptosis, and inflammation. In humans, miRNAs are also involved in immune response $(11,12)$, suggesting that they can serve as potential targets in the treatment of autoimmune diseases. The increase in the expression level of miR-155 plays an important role in the occurrence and development of RA. Wang et al. found that overexpression of miR-155 can promotes RASF proliferation and the secretion of inflammatory factors via FOXO3a (13). In addition, Inoue et al. showed that miR-182 is an important regulator of bone homeostasis and osteoclasts. In their study, the treatment of ovariectomized osteoporotic and arthritic mice with miR-182 effectively inhibited pathological bone loss and protected against excessive osteoclast proliferation (14). In this study, the expression of miR-449 in RA synovial tissue and RASFs was significantly decreased, suggesting that this miRNA may be involved in the pathogenesis of RA.

In general, miRNAs regulate the mRNA expression of their downstream target genes through targeting different sites, and play various biological functions (15). Yang et al. reported that targeting the miR-449/HDAC1 molecular axis could improve the cardiac function of elderly mice (16). Buurman et al. also evidenced the role played by miR-449/HDAC1 in the occurrence and development of liver cancer (17). In the current study, we found that the expression of miR-449 was negatively correlated with that of HDAC1. Overexpression of mir-449 in RASFs significantly inhibited the protein and mRNA expression of HDAC1, as well as inhibiting the fluorescence intensity of the HDAC1 3'-
UTR WT plasmid. These observations suggest that the miR-449/HDAC1 molecular axis also plays a role in RASFs. Subsequently, a series of reversion experiments was conducted to confirm the effects of the miR-449/HDAC1 molecular axis on RASF proliferation, invasion, and apoptosis.

RASFs in synovial tissue secrete proinflammatory factors and destructive matrix enzymes, which destroy joints (18); therefore, inhibiting the proliferation and inducing the apoptosis of these cells is extremely important in the treatment of RA. Inhibiting RASF invasion also plays a positive role in hindering the progression of RA. This study first confirmed the effects of miR-449 and its downstream target gene on HDAC1 on RASFs, and clarified the mechanism of the miR-449/HDAC1 molecular axis in the pathogenesis of RA. Although miR-449 and HDAC1 may constitute potential targets for RA treatment, further research is still needed to understand the complete mechanism. In the follow-up study, we will study the effects of the mir-449/HDAC1 molecular axis on the Wnt signaling pathway in an effort to provide a new therapeutic approach for RA treatment.

\section{Acknowledgments}

Funding: This project was supported by funding from Ningxia Natural Science Foundation Project (grant No. AAC02035 2020), Ningxia Autoimmune Disease Precision Medicine Key Laboratory (Ningxia People's Office 2020), the National Natural Science Foundation of China Regional Science Foundation Project (grant No. 81660281), the National Natural Science Foundation of China (grant No. 81260270), and the National Natural Science Foundation of China Regional Science Foundation Project (grant No. $81260460)$.

\section{Footnote}

Reporting Checklist: The authors have completed the MDAR reporting checklist. Available at https://dx.doi. org/10.21037/apm-21-1383

Data Sharing Statement: Available at https://dx.doi. org/10.21037/apm-21-1383

Conflicts of Interest: All authors have completed the ICMJE uniform disclosure form (available at https://dx.doi. org/10.21037/apm-21-1383). The authors have no conflicts of interest to declare. 
Ethical Statement: The authors are accountable for all aspects of the work in ensuring that questions related to the accuracy or integrity of any part of the work are appropriately investigated and resolved. All procedures performed in this study involving human participants were in accordance with the Declaration of Helsinki (as revised in 2013). The study was approved by the Ethics Committee of People's Hospital of Ningxia Hui Autonomous Region (No.: 2020-KY-107) and informed consent was taken from all the patients.

Open Access Statement: This is an Open Access article distributed in accordance with the Creative Commons Attribution-NonCommercial-NoDerivs 4.0 International License (CC BY-NC-ND 4.0), which permits the noncommercial replication and distribution of the article with the strict proviso that no changes or edits are made and the original work is properly cited (including links to both the formal publication through the relevant DOI and the license). See: https://creativecommons.org/licenses/by-nc-nd/4.0/.

\section{References}

1. Ngian GS. Rheumatoid arthritis. Aust Fam Physician 2010;39:626-8.

2. Balandraud N, Roudier J. Epstein-Barr virus and rheumatoid arthritis. Joint Bone Spine 2018;85:165-70.

3. Hawtree S, Muthana M, Wilkinson JM, et al. Histone deacetylase 1 regulates tissue destruction in rheumatoid arthritis. Hum Mol Genet 2015;24:5367-77.

4. Göschl L, Preglej T, Boucheron N, et al. Histone deacetylase 1 (HDAC1): A key player of T cell-mediated arthritis. J Autoimmun 2020;108:102379.

5. Hammaker D, Firestein GS. Epigenetics of inflammatory arthritis. Curr Opin Rheumatol 2018;30:188-96.

6. Evangelatos G, Fragoulis GE, Koulouri V, et al. MicroRNAs in rheumatoid arthritis: From pathogenesis to clinical impact. Autoimmun Rev 2019;18:102391.

7. Wang Y. Tripterine ameliorates monosodium urate crystal-induced gouty arthritis by altering macrophage polarization via the miR-449a/NLRP3 axis. Inflamm Res 2021;70:323-41.

8. George G, Shyni GL, Raghu KG. Current and novel therapeutic targets in the treatment of rheumatoid arthritis. Inflammopharmacology 2020;28:1457-76.
9. Huang $\mathrm{X}, \mathrm{Xu} \mathrm{J}$, Huang $\mathrm{M}$, et al. Histone deacetylase1 promotes TGF- $\beta 1$-mediated early chondrogenesis through down-regulating canonical Wnt signaling. Biochem Biophys Res Commun 2014;453:810-6.

10. Miao CG, Yang YY, He X, et al. Wnt signaling pathway in rheumatoid arthritis, with special emphasis on the different roles in synovial inflammation and bone remodeling. Cell Signal 2013;25:2069-78.

11. Salvi V, Gianello V, Tiberio L, et al. Cytokine Targeting by miRNAs in Autoimmune Diseases. Front Immunol 2019;10:15.

12. Wu H, Chen $\mathrm{Y}$, Zhu H, et al. The Pathogenic Role of Dysregulated Epigenetic Modifications in Autoimmune Diseases. Front Immunol 2019;10:2305.

13. Wang Y, Feng T, Duan S, et al. miR-155 promotes fibroblast-like synoviocyte proliferation and inflammatory cytokine secretion in rheumatoid arthritis by targeting FOXO3a. Exp Ther Med 2020;19:1288-96.

14. Inoue K, Deng Z, Chen Y, et al. Bone protection by inhibition of microRNA-182. Nat Commun 2018;9:4108.

15. Ritchie W, Rasko JE, Flamant S. MicroRNA target prediction and validation. Adv Exp Med Biol 2013;774:39-53.

16. Yang B, Zhao H, Dong R. MiR-449 improves cardiac function by regulating HDAC1 and cTnI. Eur Rev Med Pharmacol Sci 2020;24:12827-35.

17. Buurman R, Gürlevik E, Schäffer V, et al. Histone deacetylases activate hepatocyte growth factor signaling by repressing microRNA-449 in hepatocellular carcinoma cells. Gastroenterology 2012;143:811-820.e15.

18. Neumann E, Lefèvre $S$, Zimmermann B, et al. Rheumatoid arthritis progression mediated by activated synovial fibroblasts. Trends Mol Med 2010;16:458-68. Erratum in: Trends Mol Med 2011;17:118.

(English Language Editor: J. Reynolds)

Cite this article as: Guo J, Cao X, Zhao W, Zhu H, Ma X, Hao C, Wu L, Zhang M, Yang Y, Zhao J, Chen K, Yin Z. MicroRNA-449 targets histone deacetylase 1 to regulate the proliferation, invasion, and apoptosis of synovial fibroblasts in rheumatoid arthritis. Ann Palliat Med 2021;10(7):7960-7969. doi: 10.21037/apm-21-1383 